\title{
Illustrations of
}

Plant Microfossils

From the Yazoo Clay

(Jackson Group,Upper Eocene) Mississippi

GEOLOGICAL SURVEY PROFESSIONAL PAPER 643-E 


\section{Illustrations of}

Plant Microfossils

From the Yazoo Clay

(Jackson Group,Upper Eocene) Mississippi

By ROBERT H. TSCHUDY and SHARON D. VAN LOENEN

CONTRIBUTIONS TO PALEONTOLOGY

GEOLOGICAL SURVEY PROFESIONAL PAPER 643-E

An assemblage of Yazoo Clay palynomorphs is

illustrated and discussed briefly; tentative

taxonomic identifications are made

to the generic level

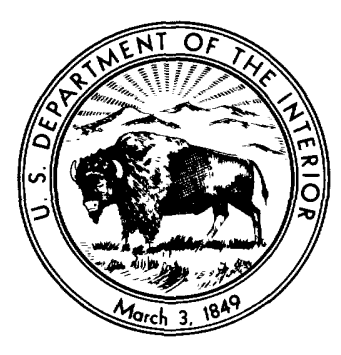

UNITED STATES GOVERNMENT PRINTING OFFICE, WASHINGTON : 1970 


\section{UNITED STATES DEPARTMENT OF THE INTERIOR \\ WALTER J. HICKEL, Secretary \\ GEOLOGICAL SURVEY \\ William T. Pecora, Director}

For sale by the Superintendent of Documents, U.S. Government Printing Office

Washington, D.C. 20402 - Price 50 cents (paper cover) 


\section{CONTENTS}

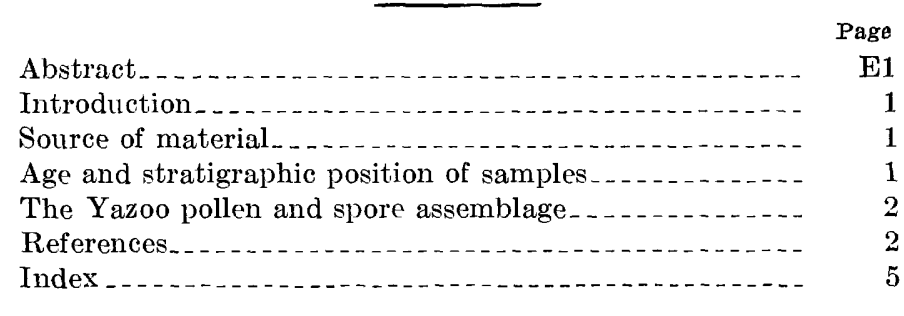

I L L U S T R A T I O N S

[Plates follow index]

Plates 1-5. Yazoo pollen and spores.

E2

Figure 1. Index map showing sample localities 



\title{
CONTRIBUTIONS TO PALEONTOLOGY
}

\section{ILLUSTRATIONS OF PLANT MIGROFOSSILS FROM THE YAZOO GLAY (JAGKSON GROUP, UPPER EOGENE), MISSISSIPPI}

\author{
By Robert H. Tschudy and Sharon D. Van Loenen
}

\begin{abstract}
This publication illustrates a pollen and spore assemblage from the marine Yazoo Clay of Mississippi. The late Eocene age of the formation is well established by mollusks and Foraminifera. Figures on each plate are supplied with provisional generic identification. Many of the species have not previously been illustrated.
\end{abstract}

\section{INTRODUCTION}

U.S. Geological Surrey palynologists have accumulated many palynological assemblages of diverse ages from type or well-documented sample localities. We anticipate that this material eventually will be the subject of detailed taxonomic treatment. The present publication is intended as a means of presenting some of these data in a condensed, preliminary form.

The usefulness of palynolngical assemblages for stratigraphic determinations and for laboratory reference has been amply demonstrated in a series of papers published by the Canadian Geological Survey, as listed by Barss (1967). These assemblages serve to make information immediately available that would otherwise be unarailable until such time as a full systematic treatment could be accomplished. This publication, like the papers of the Canadian Geological Survey, is designed primarily for laboratory reference and is in no way intended as a substitute for thorough taxonomic treatment. It is a tentative guide to the preliminary identification of the more common species present in the assemblage. No attempt was made to record all the rare species that were present in the material.

\section{SOURCE OF MATERIAL}

The Yazoo Clay, named from exposures in the bluff of the Yazoo River at Yazoo City, Miss., is a marine unit consisting of blue-green to blue-gray calcareous, glauconitic, fossiliferous, silty clay and clay. It has an arerage thickness of about 450 feet and attains a maximum thickness of about 525 feet in southwestern Hinds
County, Miss. (Moore, 1965). The Yazoo outcrop belt forms what is known as the Jackson Prairie in Mississippi. It also crops out in Alabama and Louisiana. Priddy (1960) stated: "Despite the broad belt of outcrops of the Yazoo Formation, good exposures of the Yazoo Clay are rare." We were fortunate in obtaining good sample material from the following localities:

Core hole at the type locality, SE1/4 SW1/4 SE1/4 sec. 32, T. 12 N., R. 2 W., Yazoo County, Miss.

Core depth $6 \pm$ feet, USGS Paleobotanical locality D3697-B.

Core depth $1+0$ feet, USGS Paleobotanical locality D3697-A. (See Moore and others, 1964, p. 13.)

Core hole $\mathrm{AF}-10,25$ feet north of east-west gravel road in SW1/4 SE1/4 NW1/4 sec. 5, T. 7 N., R. 1 W., Hinds County, Miss.

Core depth 26 feet, USGS Paleobotanical locality D3698-B.

Core depth 44 feet, USGS Paleobotanical locality D3698-A.

The above samples were supplied through the courtesy of the Mississippi Geological Survey.

Jackson Ready-Mix Concrete-Light Aggregate Quarry, SW1 $1 / 4$ NE1/4NE1/4 sec. 36, T. 7 N., R. 1 W., Hinds County, 7 miles west-southwest of Madison, Miss. (Priddy, 1960, p. 40, and Moore, 1965, pl. 1).

Surface sample 10 feet below top, USGS Paleobotanical locality D3699-B.

Surface sample 30 feet below top, USGS Paleobotanical locality D3699-A.

The location of these samples is shown in figure 1.

\section{AGE AND STRATIGRAPHIC POSITION OF THE SAMPLES}

The Jackson Group in Mississippi consists of the Moodys Branch Formation and the overlying Yazoo Clay. The Jackson Group was first differentiated from other Eocene rocks by Conrad (1855), who also de- 
scribed the molluscan fossils. Harris and Palmer (1945), in a monograph on the Jackson mollusks, provided additional data, which support a late Eocene age for the Jackson Group. Foraminiferal and ostracode microfossil evidence also supports a late Eocene age for the Yazoo Clay and its equivalents (Deboo, 1965). Apparently there is no question concerning the age of this unit.

The Yazoo Clay, according to Mellen (1940), conformably overlies the Moodys Branch Formation and conformably underlies the Forest Hill Sand. The Forest Hill is of Oligocene age; however, Priddy (1960) stated: $" * * *$ the Yazoo-Forest Hill contact is definitely recognizable in a few places but indefinite in most. There can be no doubt as to the disconformable relation in Test Holes 7 and 8 where non-marine strata overlie calcareous marine clays. However, on the surface the rontact is difficult to find where (1) non-marine Forest Hill clays lie on Yazoo (lays which had been leached before Forest Hill deposition, and (2) where aprons of rain-washed Forest Hill sands or silts hide the contact

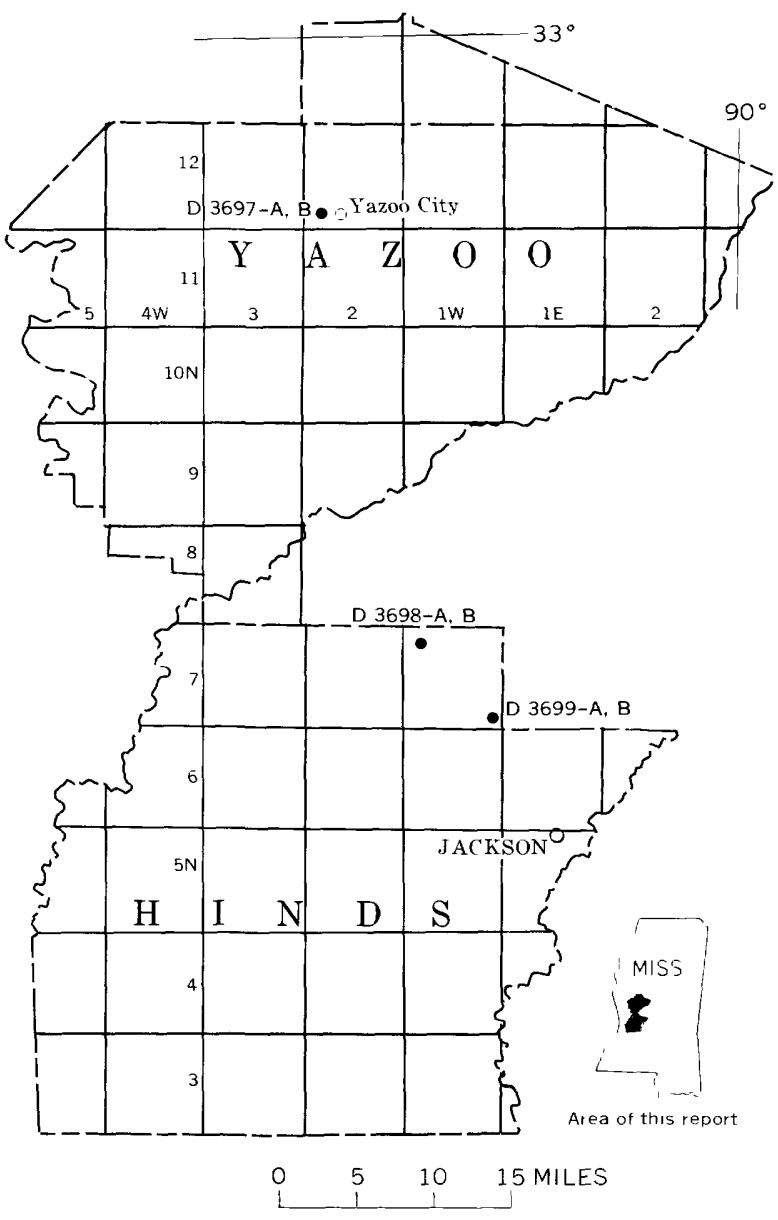

Figure 1.-Index map showing sample localities in Yazoo and Hinds Counties, Miss. in gullied areas." The conformable-gradational Moodys Branch-Yazoo contact was also indicated by Moore (1965).

The stratigraphic position of the samples is summarized as follows :

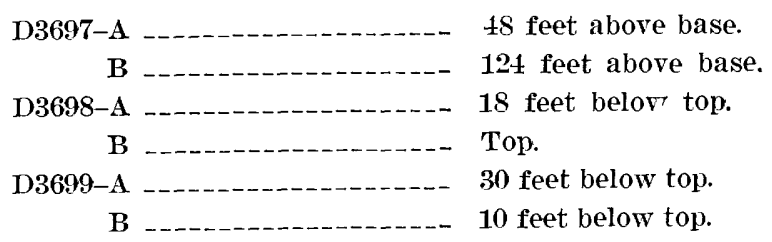

THE YAZOO POLLEN AND SPORE ASSEMBLAGE

Pollen and spore species found in the Yazoo material are shown on plates 1-5. Samples D3697-B and D3699A yielded the most diverse assemblages and the best preserved specimens; consequently, most of the photographed specimens are from these two samples. All the Yazoo samples yielded abundant marine hystrichospheres and dinoflagellates. These, however, are not included on the plates.

The generic identifications given in the plate descriptions are believed to be reliable but are subject to the constant revision that palynological no'nenclature is undergoing at present. With few exceptions, no attempt is made to indicate species.

The plate descriptions include the locality number and a number in parentheses. The latter is the number of the slide on which that particular specimen is found. The slides and the microscope coordinates for each of the photographed specimens are on file in the USGS Denver Palynological Laboratory.

\section{REFERENCES}

Anderson, R. Y., 1960, Cretaceous-Tertiary palynology. eastern side of the San Juan Basin, New Mexico: New Mexico Bur. Mines and Mineral Resources Mem. 6, $58 \mathrm{p}$.

Barss, M. S., 1967, Illustrations of Canadian fos $>i l s-$ Carboniferous and Permian spores of Canada: Canada Geol. Survey Paper 67-11, $94 \mathrm{p}$.

Conrad, T. A., 1855, Observations on the Eocere deposit of Jackson, Mississippi, with descriptions of thirty-four new species of shells and corals: Acad. Nat. Sci. Philadelphia Proc. 7, p. 257-263 (reprinted, 1939, Am. Paleontology, v. 24 , no. 86,19 p.).

Deboo, P. B., 1965, Biostratigraphic correlation of the type Shubuta Member of the Yazoo Clay and Red Bluff Clay with their equivalents in southwestern Alabama: A labama Geol. Survey Bull. 80, 84 p.

Engelhardt, D. W., 196t, Plant microfossils fror the Eocene Cockfield Formation, Hinds County, Mississippi: Mississippi Geol. Econ. and Topog. Survey Bull. 104, p. 65-95.

Harris, G. D., and Palmer, K. V., 1946-47, The Mollusea of the Jackson Eocene of the Mississippi Embayment (Sabine River to the Alabama River): Bull. Am. Paleontology, v. 30, no. 117, pt. 1, Bivales, 206 p.; pt. 2. Univalves p. 207-563. 
Kedves, Miklos, 1960. Études palynologiques dans le bassin de Dorog: Pollen et Spores, r. 2, no. 1. p. 89-118.

Mellen, F. F., 1940, Yazoo County mineral resources: Mississippi State Geol. Surver Bull. 39, 132 p.

Munre, T. H., 196.T, Hinds County geology: Mississippi Geol. Econ. and Topog. Survey Bull. 105, p. 21-145.

Moore, W. H., Parks, W. S., and Kern, M. K., 196t, Trpe localities sampling program: Mississippi Geol. Econ. and Topog. Survey Bull. 104, p. 7-32.

P'otonié. Robert, 1934, Zur Mikrohotanik der Kohlen und ihrer Verwandten. II, Zur Mikrobotanik des eocänen Humodils des Geiseltals: Preuss, Geol. Landesanstalt. Inst. Paläeobot. und Petrographie Brennsteine, Arb., v. 4, p. 25-125.

Potonié, Robert. Thomson, P. W., and Thiergart, Friedrich, 1951. Zur Nomenklatur und Klassifikation der neogenen Sporomorphae (Pollen und Sporen): Geol. Jahrb., v. 65, p. $35-69$.

Priddy, R. R., 1960, Madison County geology: Mississipui State Geol. Survey Bull. 88, 123 1.
Raatz, G. V., 1937, Mikrobotanisch-stratigraphische Untersuchung der Braunkohle des muskauer Bogens: Preuss. Geol. Landesanstalt, Abh., Neue Folge, 183, 48 p.

Steeves, M. W., and Barghoorn, E. S., 1959. The pollen of Ephedra: Harvard Univ., Arnold Arboretum Jour., v. 40 , no. 3, p. $221-255$.

Stover, L. E., Elsik, W. C., and Fairchild, W. W., 1966. New genera and species of early Tertiary palynomorphs from Gulf Coast: Kansas Iniv. Paleont. Contr., Paper 5, p. 1-11.

Thomson, P. W., and Pflug, Hans, 1953, Pollen und Sporen des mitteleuropäischen Tertiärs: Palaeontographica, sec. Б., v. 94. $138 \mathrm{p}$.

Tsukada, Matsuo, 1964, Pollen morphology and identificetion III-Modern and fossil tropical pollen with emphasis on Bomluacaceae: Pollen et Spores, v. 6, no. 2, p. 393-462

Wolff. Herbert, 1934, Mikrofossilien des pliocänen Humodils der Grube Freigericht bei Dettlingen a. M. und Vergleich mit älteren Schichten des Tertiärs sowie posttertiären Ablagerungen: Preuss. Geol. Landesanstalt, Inst. Paläeobot. und Petrographie Brennsteine, Arb., v. 5, p. 55-86. 



\section{INDEX}

[Italic numbers indicate major references]

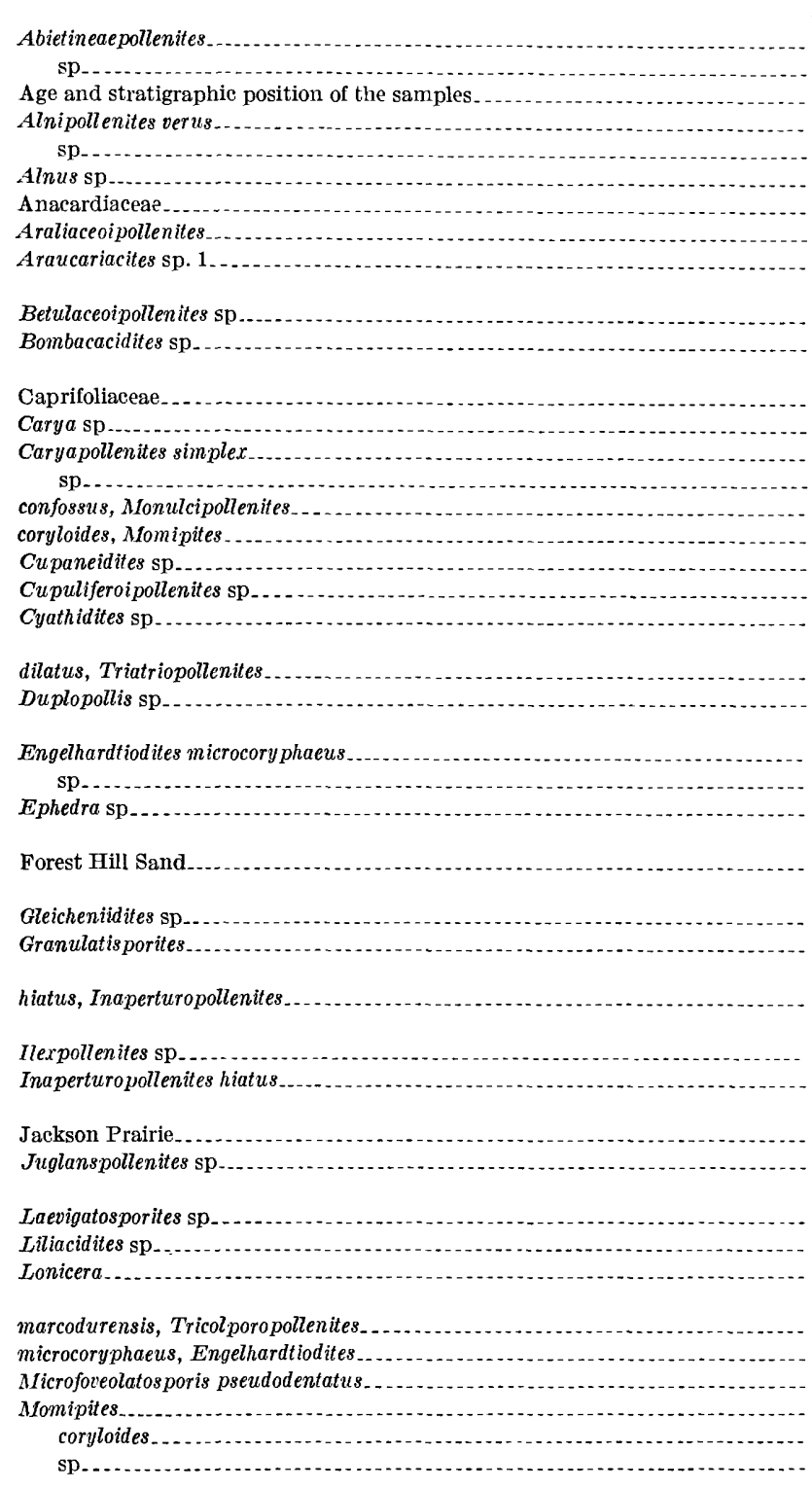

Page pollenites nupharoides ....

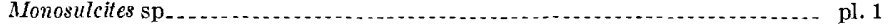

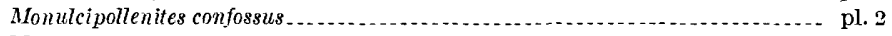

Moodys Branch Formation......... E1

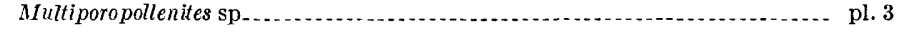

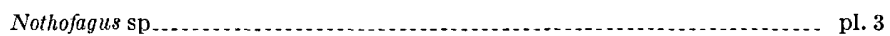

nu pharoides, Monocol popollenites........................................... pl. 2

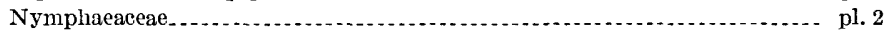

Osmundacidites

Paliurus triplicatus.

Parthenocissus............. 5

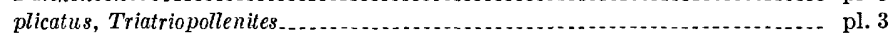

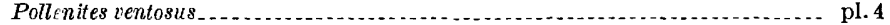

Polyporopollenites sp............... 3

pseudodentatus, Microfoveolatosporis

Rhoipites sp...............

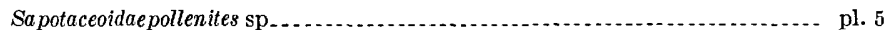

simplex, Caryapollenites.....

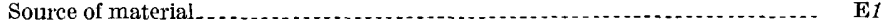

Spondias

Stratigraphic position and age of the samples.

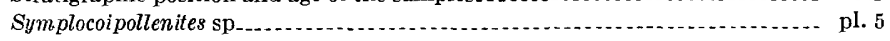

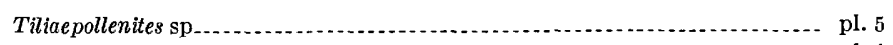

Triangular spore

Triatriopollenites dilatus.................................................... pl. 3

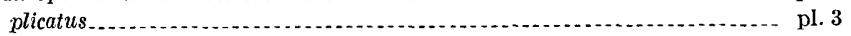

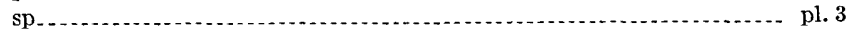

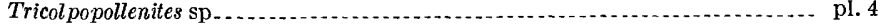

Tricol porites sp...........................................

Tricolporopollenites marcodurensis.

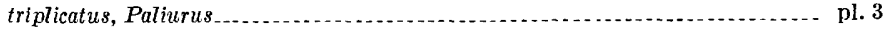

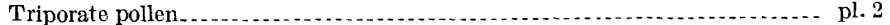

Triporopollenites sp..

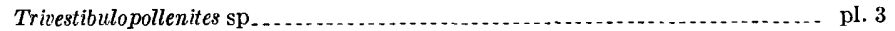

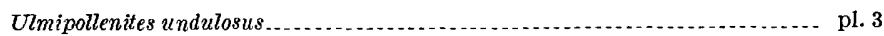

sp.

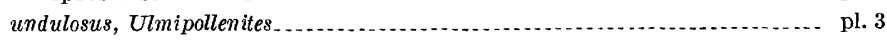

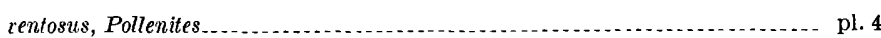

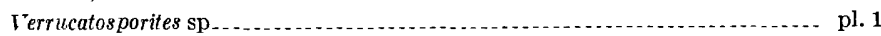

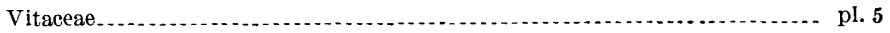

Yazoo pollen and spore assemblage ................................... E2 
PLATES 1-5 


\section{PLATE 1}

[Magnification $\times 1,000$ unless otherwise indicated]

Locality and slide Nos.

FIGURE 1. Laevigatosporites sp_....... D3699-A (3)

2. Verrucatosporites sp_...

3. Microfoveolatosporis ef. $M$. pseudodentatus Engelhardt 1964_... D3699-A (3)

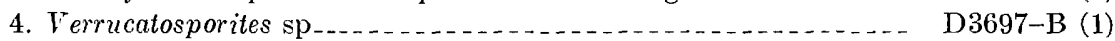

5. Verrucatosporites sp_..._._.

6. Verrucatosporites sp..._.

7. Cyathidites sp.......... D3699-A (3)

8a. Osmundacidites, high focus showing laesurae._._......... D3697-B (1)

8 b. Same specimen, low focus.

9. Monosulcites sp..... D3699-A (1)

10. Monosulcites sp_... D3699-A (3)

11. Gleicheniidites sp_._._._. D3699-A (1)

12. Triangular spore of Granulatisporites type._._........... D3699-A (4)

13. Ephedra sp. (type A of Steeves and Barghoorn 1959) _....... D3697-B (1)

14. Monosulcites sp. (probably same sp. as fig. 10) _......... D3697-B (1)

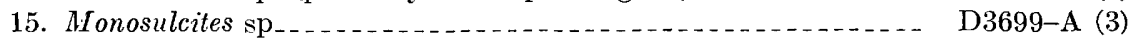

16. Liliacidites $\mathrm{sp} \ldots \ldots \ldots$ D3697-B (1)

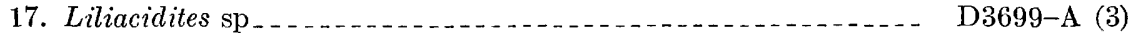

18. Liliacidites sp. (same species as fig. 17, magnified $\times 2,000$ to show structure of reticulum)

D3699-A (1) 

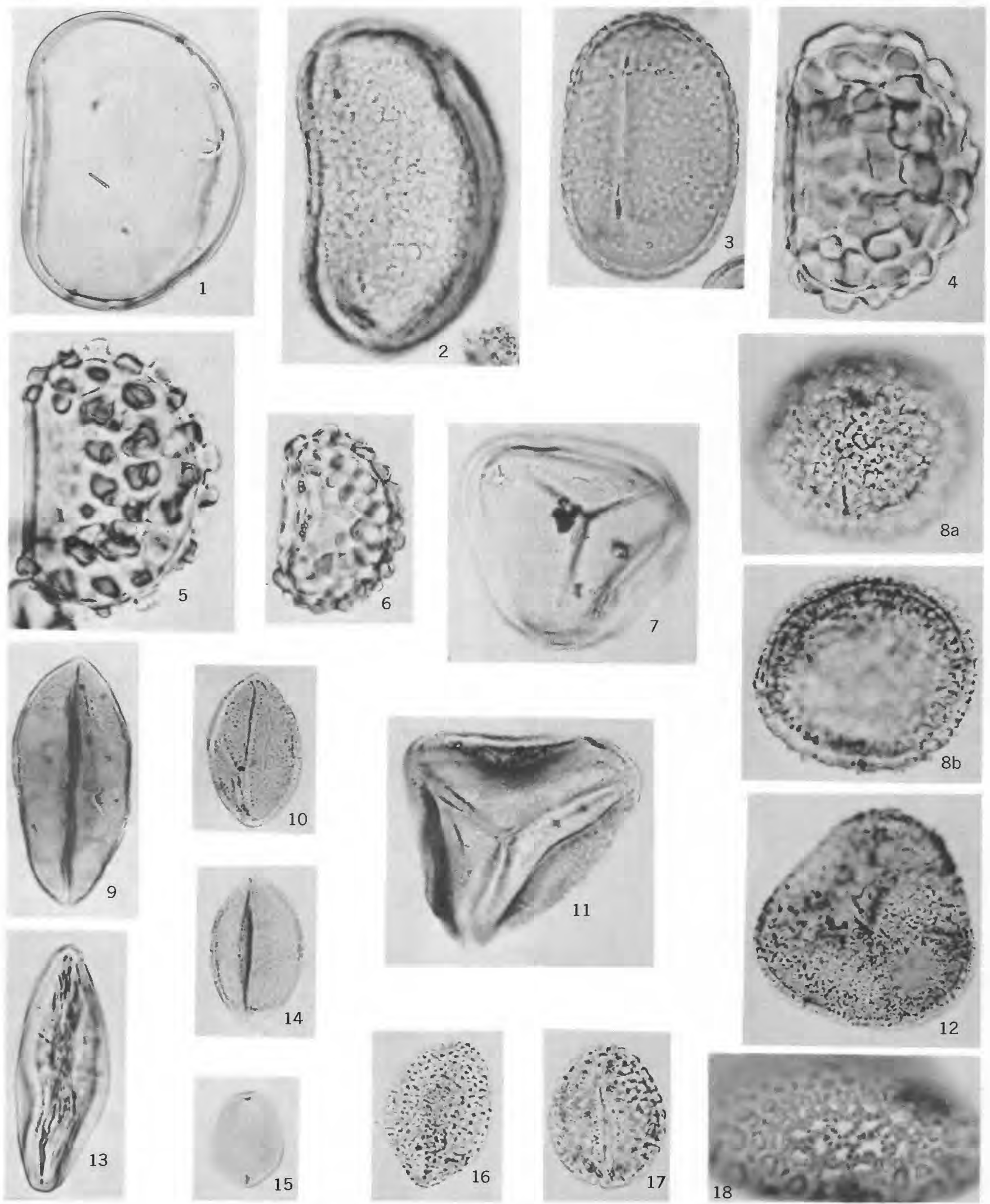

YAZOO POLLEN AND SPORES 


\section{PLATE 2}

$[$ Magnification $\times 1,000]$

Locality and slide Nos.

Figure 1. Nymphaeaceae (see Monocolpopollenites nupharoides Kedves

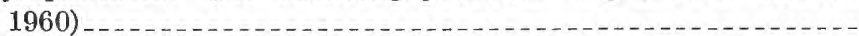

2a. Nymphaeaceae, same species as fig. 1, low focus_............

D3697-B (1)

2b. Nymphaeaceae, same specimen as fig. 2a, high focus.

3. ?Araucariacites sp. 1 D3699-A (3)

4. Abietineaepollenites sp. (Diploxylon type)

5. Inaperturopollenites cf. I. hiatus (R. Potonié) Thomson and

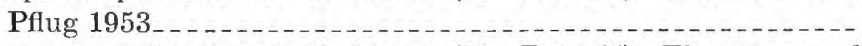

6. Inaperturopollenites cf. I. hiatus (R. Potonié) Thomson and Pflug 1953

D3699-A (3)

D3697-B (1)

D3699-A (3)

7. Abietineaepollenites sp. (Diploxylon type)

8. Abietineaepollenites sp. (Diploxylon type)

9. Abietineaepollenites sp. (Diploxylon type)

10. Triporate pollen

D3697-B (2)

D3699-A (4)

D3699-A (4)

D3697-B (1)

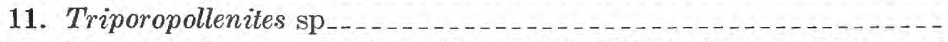

D3697-B (2)

12a. Monulcipollenites cf. $M$. confossus Fairchild, in Stover, Elsik, and Fairchild 1966. High focus

12b. Same specimen as fig. 12a. Low focus.

13. Triporopollenites sp_...

14. Abietineaepollenites (Diploxylon type)

15. ?Momipites sp. (See $M$. coryloides Wode. 1933, in Englehardt 1964)

D3699-A (3)

16. Triporopollenites $\mathrm{sp}$

D3699-A (3)

D3697-B (1)

D3697-B (1)

D3699-A (4) 

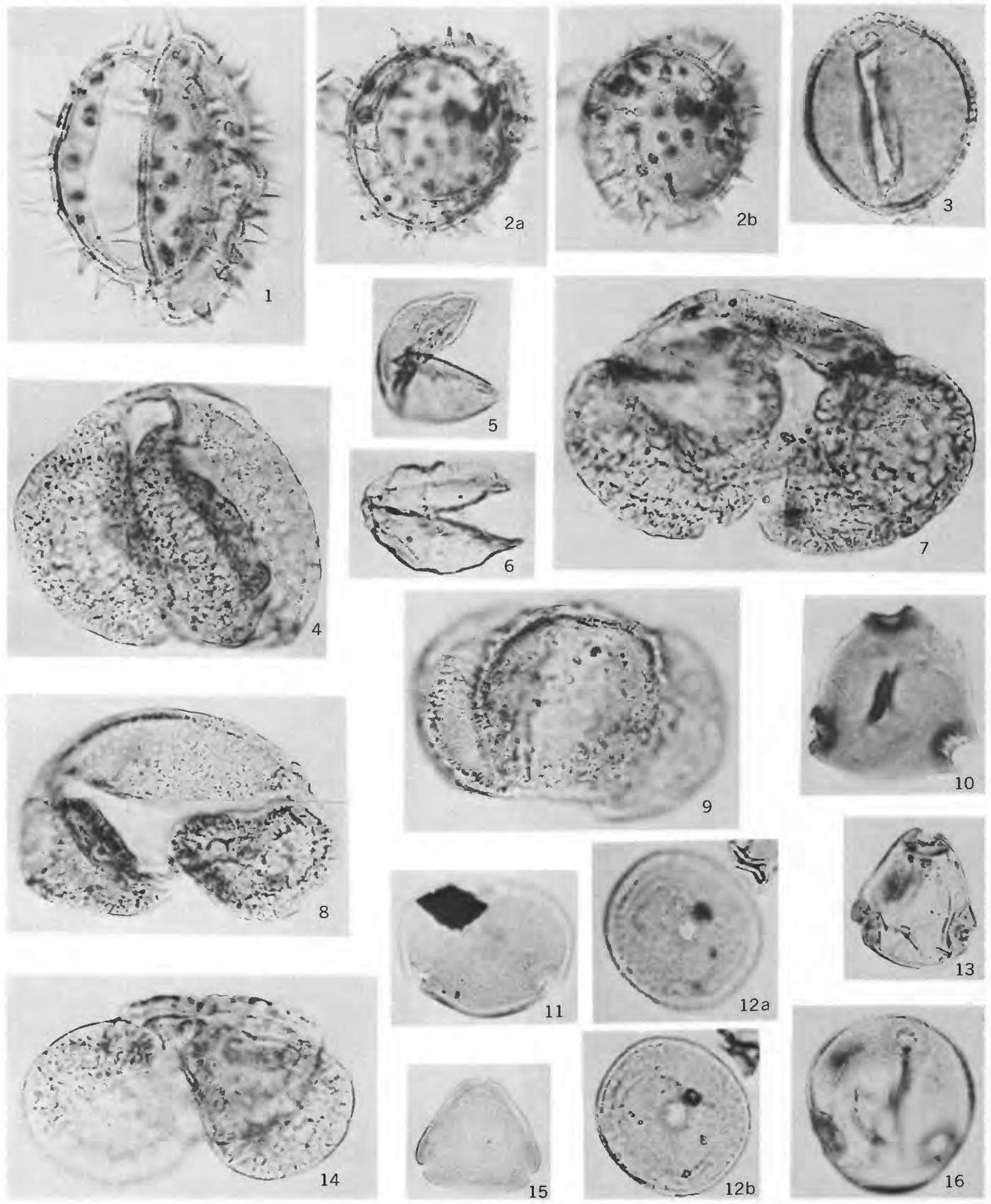

YAZOO POLLEN AND SPORES 


\section{PLATE 3}

Figure 1. Triatriopollenites $\mathrm{sp}$

D3697-B (2)

2. Triatriopollenites sp

D3699-A (3)

3. ?Engelhardtiodites cf. E. microcoryphaeus (Potonié) Potonié, Thomson, and Thiergart 1950. (See Engelhardt 1964.)

D3699-A (3)

4. Triatriopollenites $\mathrm{sp}$

D3699-A (3)

5. Engelhardtioidites sp...

D3699-A (3)

6. Triatriopollenites of. $T$. dilatus Fairchild in Stover, Elsik, and Fairchild 1966

D3699-A (4)

7. Triatriopollenites sp

8. ?Betulaceoipollenites $\mathrm{sp}_{\ldots}$

9. Triatriopollenites sp. cf. T. plicatus Thomson and Pflug 1953_-

10. Carya sp. or Caryapollenites $\mathrm{sp}$

11. Carya sp. or Caryapollenites sp. cf. C. simplex (Potonié) Raatz 1937

D3699-A (3)

D3699-A (3)

D3699-A (1)

D3699-A (3)

12a. Carya sp. or Caryapollenites sp. Low focus_.............

12b. Same specimen as fig. 12a. High focus.

13. Triporopollenites sp.

14. Triatriopollenites $\mathrm{sp} \ldots$

15. Triporopollenites $\mathrm{sp} \ldots$

16. Ulmipollenites $\mathrm{sp}_{-}$

17. Polyporopollenites sp. (?four-pored Caryapollenites) _..........

18. Alnus sp. or Alnipollenites sp. cf. A. verus (R. Potonié) ex. R. Potonié 1934

19. Trivestibulopollenites sp. cf. Paliurus triplicatus Anderson 1960_

20. Alnus sp. or Alnipollenites sp.

21. Polyporopollenites sp. (cf. pl. 2, fig. 15 ?Momipites)

22. Ulmipollenites $\mathrm{sp}$

23. ?Aff. Nothofagus $\mathrm{sp}_{\ldots}$

24. Multiporopollenites $\mathrm{sp}_{\ldots} \ldots$

25. Ulmipollenites sp. ef. $U$. undulosus Wolff 1934 (see Engelhardt 1964)

D3697-B (1)

D3699-A (1)

D3699-A (3)

D3699-A (1)

D3699-A (3)

D3699-A (1)

D3699-A (3)

D3699-A (3)

D3697-B (1)

D3697-B (2)

D3699-A (3)

D3699-A (3)

D3697-B (2)

D3697-B (1)

26. Alnus sp. or Alnipollenites sp

27. ?Aff. Nothofagus sp

D3699-A (3)

28. ?Aff. Nothofagus sp

D3697-B (1)

D3699-A (3)

29. Juglanspollenites sp. _..

30. Juglanspollenites $\mathrm{sp}_{\ldots} \ldots \ldots \ldots$

31. Multiporopollenites sp__._._.

32. Multiporopollenites $\mathrm{sp}_{\ldots} \ldots \ldots$

33. Multiporopollenites $\mathrm{sp}$ 
GEOLOGICAL SURVEY
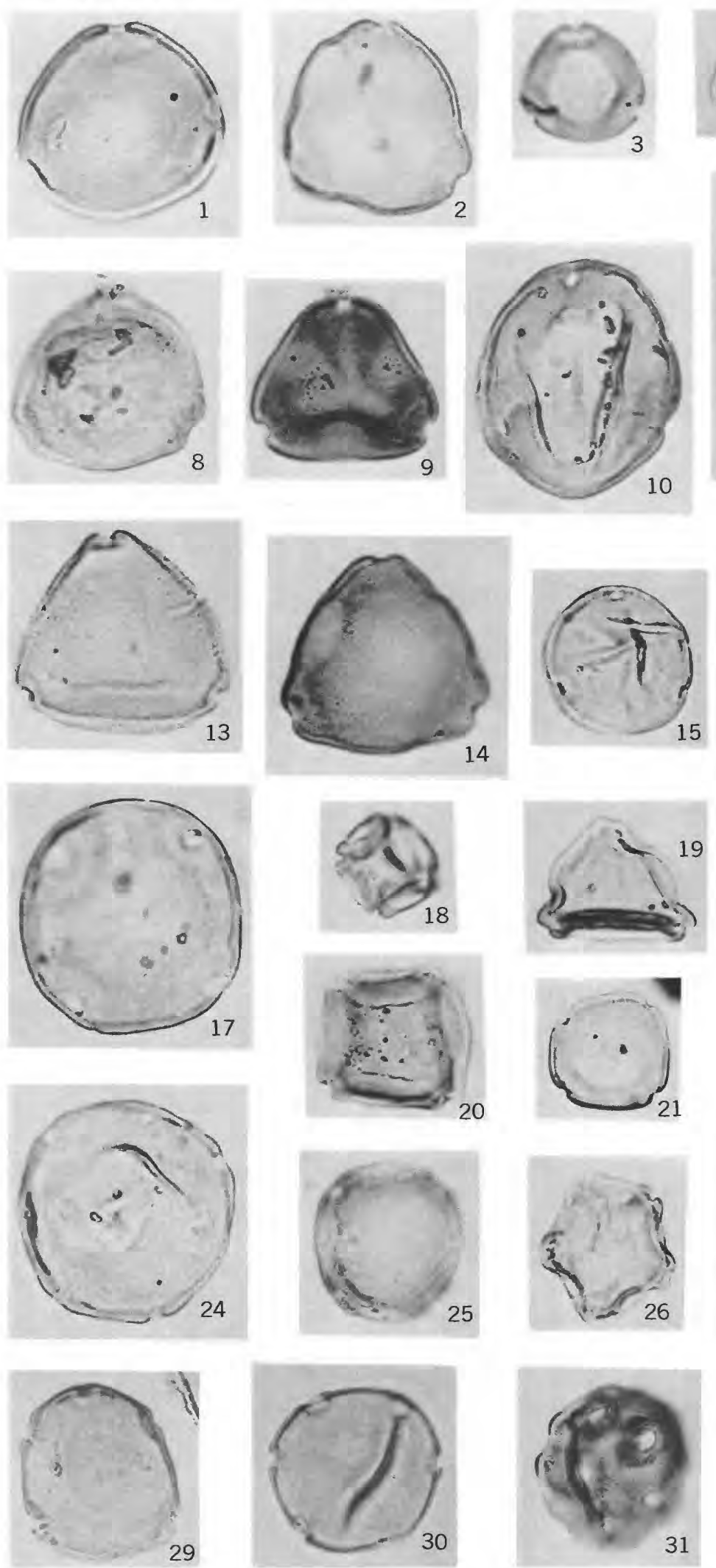
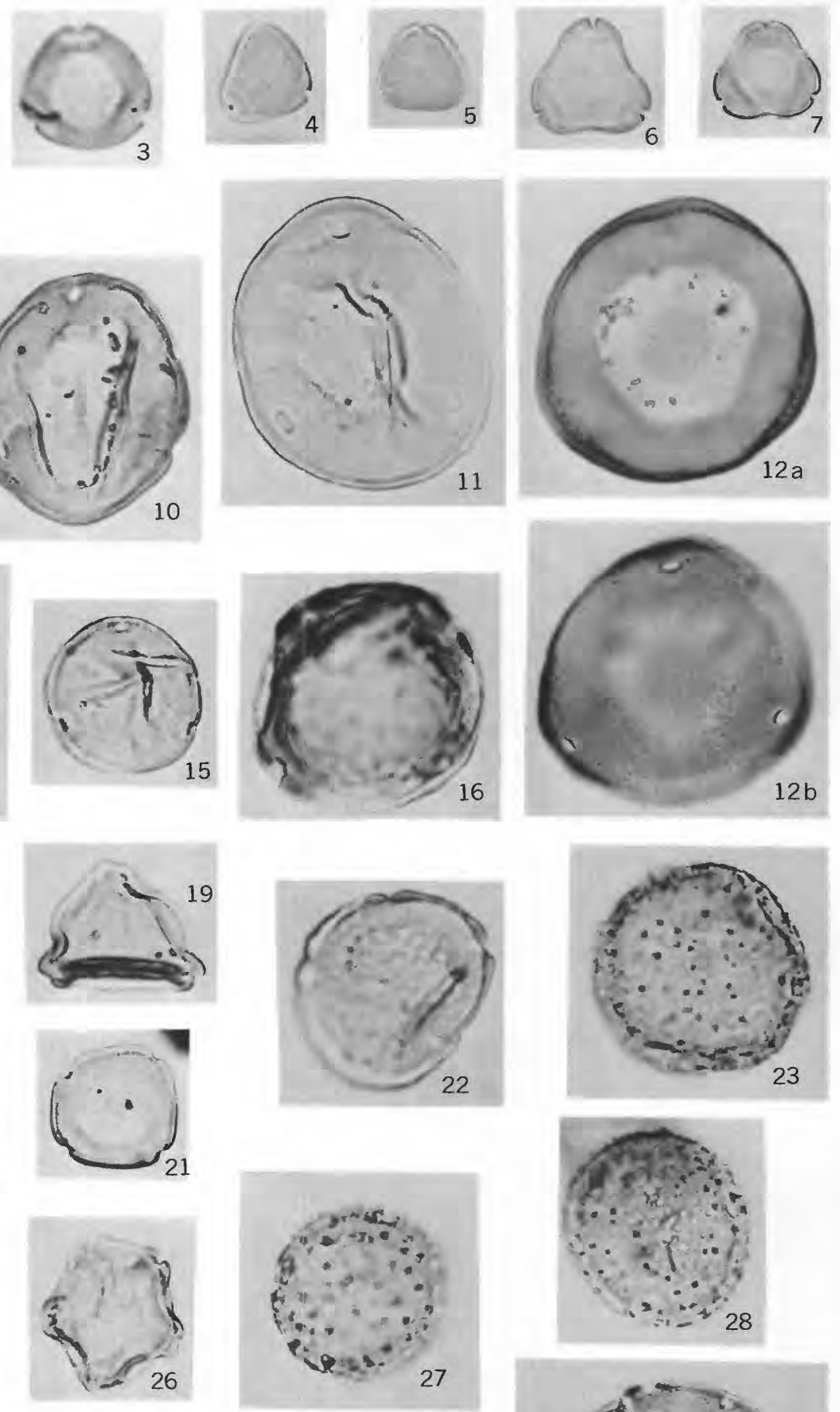

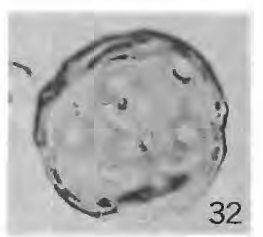

32
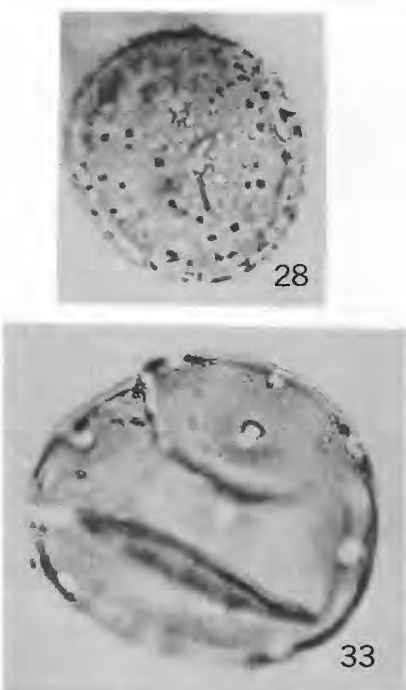

YAZOO POLLEN AND SPORES 


\section{PLATE 4}

[Magnification $\times 1,000$ ]

Locality and slide Nos.

Figure

1. Tricolpopollenites $\mathrm{sp}$

D3699-A (3)

2. Tricolpopollenites $\mathrm{sp}$

D3697-B (1)

3. Tricolpopollenites sp

4. Tricolpopollenites $\mathrm{sp}$

Tricolpopollenites $\mathrm{sp}$

7. Tricolpopollenites $\mathrm{sp}$

8. Tricolpopollenites $\mathrm{sp}$

9. Tricolpopollenites sp.

10. Tricolpopollenites sp. Same species as fig. 6_.............

11. Tricolpopollenites $\mathrm{sp}$

12. Tricolpopollenites $\mathrm{sp}$

13a. Tricolpopollenites sp. High focus _......................

13b. Same specimen as fig. 13a. Low focus.

14. Tricolpopollenites $\mathrm{sp}$

15. Cupuliferoipollenites $\mathrm{sp}$

16. Cupuliferoipollenites $\mathrm{sp}$

17. Tricolpopollenites sp. aff. Caprifoliaceae cf. Lonicera

18. Ilexpollenites $\mathrm{sp}$

19. Ilexpollenites $\mathrm{sp}_{\ldots}$

20a. Tricolpopollenites sp. Low focus

20b. Same specimen as fig. 20a. High foeus.

21a. Rhoipites sp. High focus

21b. Same specimen as fig. 21a. Low focus

22a. Tricolporites sp. (?Araliaceoipollenites) Low focus

22b. Same specimen as fig. 22a. High focus.

23. Cupaneidites sp

24. Cupaneidites $\mathrm{sp}_{\text {....... }}$

25. Duplopollis sp

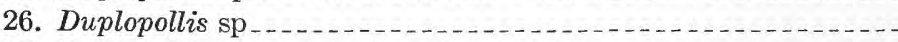

27. Duplopollis $\mathrm{sp}_{\ldots}$

28a. Tricolporites sp. High focus

28b. Same specimen as fig. 28a. Low focus.

29. Tricolporites sp. (?Anacarediacea of. Spondias. See Tsukada 1964.)

30. Tricolporites sp. (cf. Pollenites ventosus Potonić 1934)

31. Tricolporites $\mathrm{sp}$

32. Tricolporites $\mathrm{sp}$

D3697-B (1)

D3697-B (1)

D3697-B (1)

D3699-A (4)

D3697-B (1)

D3699-A (3)

D3697-B (2)

D3699-A (3)

D3697-B (2)

D3697-B (2)

D3697-B (1)

D3699-A (3)

D3697-B (1)

D3697-B (1)

D3699-B (4)

D3697-B (1)

D3699-A (3)

D3697-B (1)

D3699-A (3)

D3697-B (1)

D3699-A (3)

D3699-A (3)

D3699-A (1)

D3699-A (4)

D3699-A (4)

D3697-B (1)

D3699-A (3)

D3699-A (3)

D3697-B (2)

D3699-A (3) 

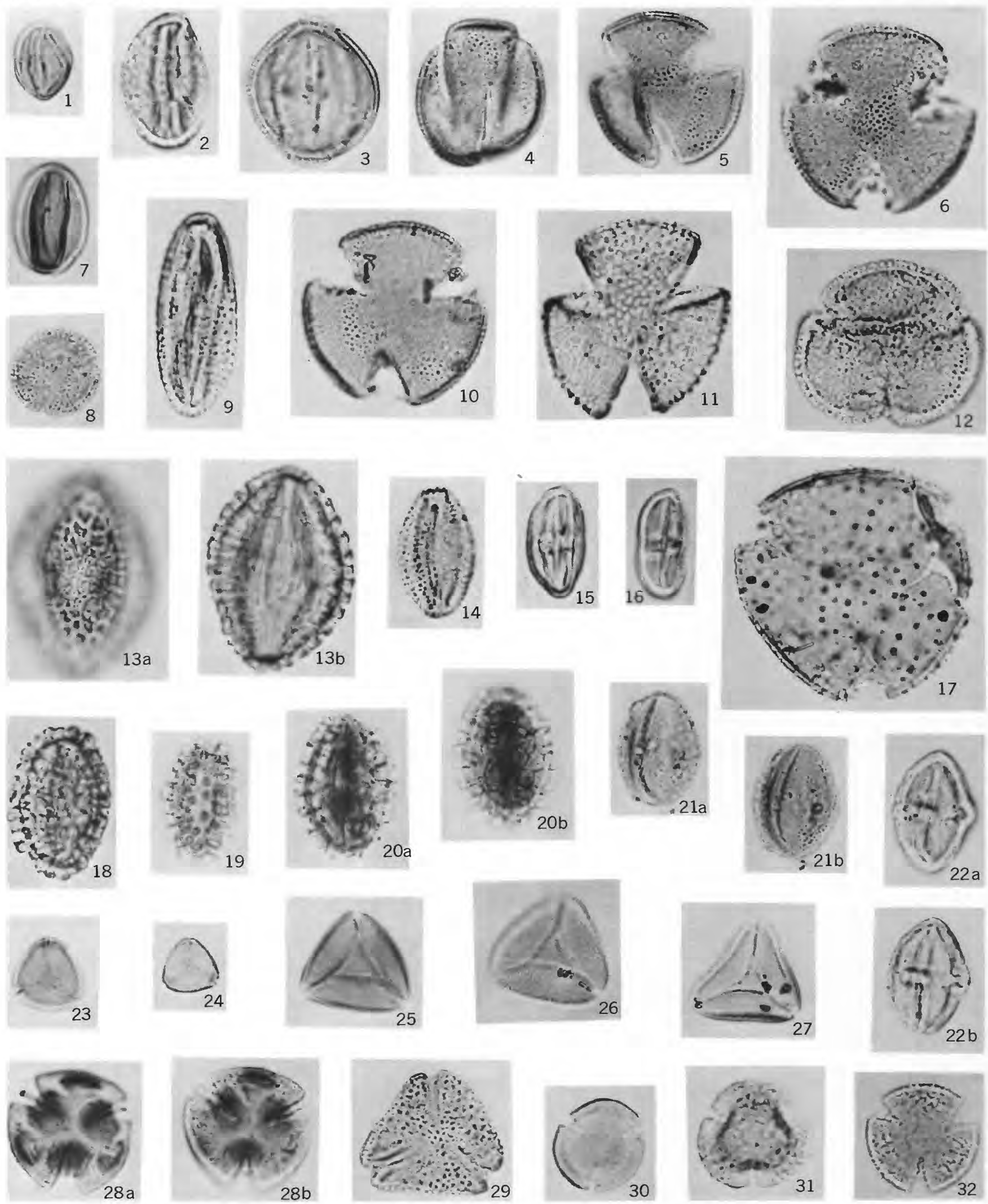

YAZOO POLLEN AND SPORES
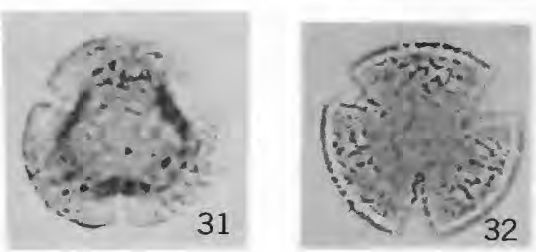


\section{PLATE 5}

[Magnification $\times 1,000]$

Locality and stide Nos.

Figure 1. Tricolporites sp. (See Tricolporopollenites marcodurensis Thomson and Pflug 1953; cf. Vitaceae, Parthenocissus.)

D3697-B (1)

2a. Rhoipites sp. High focus. . . . . . . .

2b. Same specimen as fig. 2a. Low focus.

3. Tricolporites $\mathrm{sp}$

D3699-A (3)

4. Sapotaceoidaepollenites $\mathrm{sp}$

5. Tricolporites sp

6a. Symplocoipollenites sp. Low focus

6b. Same specimen as fig. 6a. High focus.

7a. Sapotacєoidaepollenites sp. High focus

7b. Same specimen as fig. 7a. Low focus.

8a. Sapotaceoidaepollenites sp. High focus

8 b. Same specimen as fig. 8 a. Low focus.

9. Symplocoipollenites $\mathrm{sp}$

D3699-A (4)

D3697-B (1)

D3699-A (3)

D3699-A (3)

10. Symplocoipollenites sp

11a. Tiliaepollenites sp. High focus---ne--

11b. Same specimen as fig. 11a. Low focus.

12. Symplocoipollenites $\mathrm{sp}$

13. Tiliaepollenites sp.

D3699-A (3)

15. Tiliaepollenites sp. Probably the same species as fig. 14, but possessing four apertures _...

16a. Tiliaepollenites sp. Low focus _...

16b. Same specimen as fig. 16a. High focus.

17. Bombacacidites $\mathrm{sp}$

18. Bombacacidites $\mathrm{sp}$

19. Bombacacidites $\mathrm{sp}_{\ldots} \ldots$

D3697-B (1)

D3697-B (1)

D3697-B (1)

D3699-A (3)

D3699-A (1)

D3699-A (4)

D3699-A (3)

D3699-A (3)

D3699-A (4)

D3699-A (4)

D3699-A (4)

D3697-B (2) 
GEOLOGICAL SURVEY

PROFESSIONAL PAPER 643-E PLATE 5
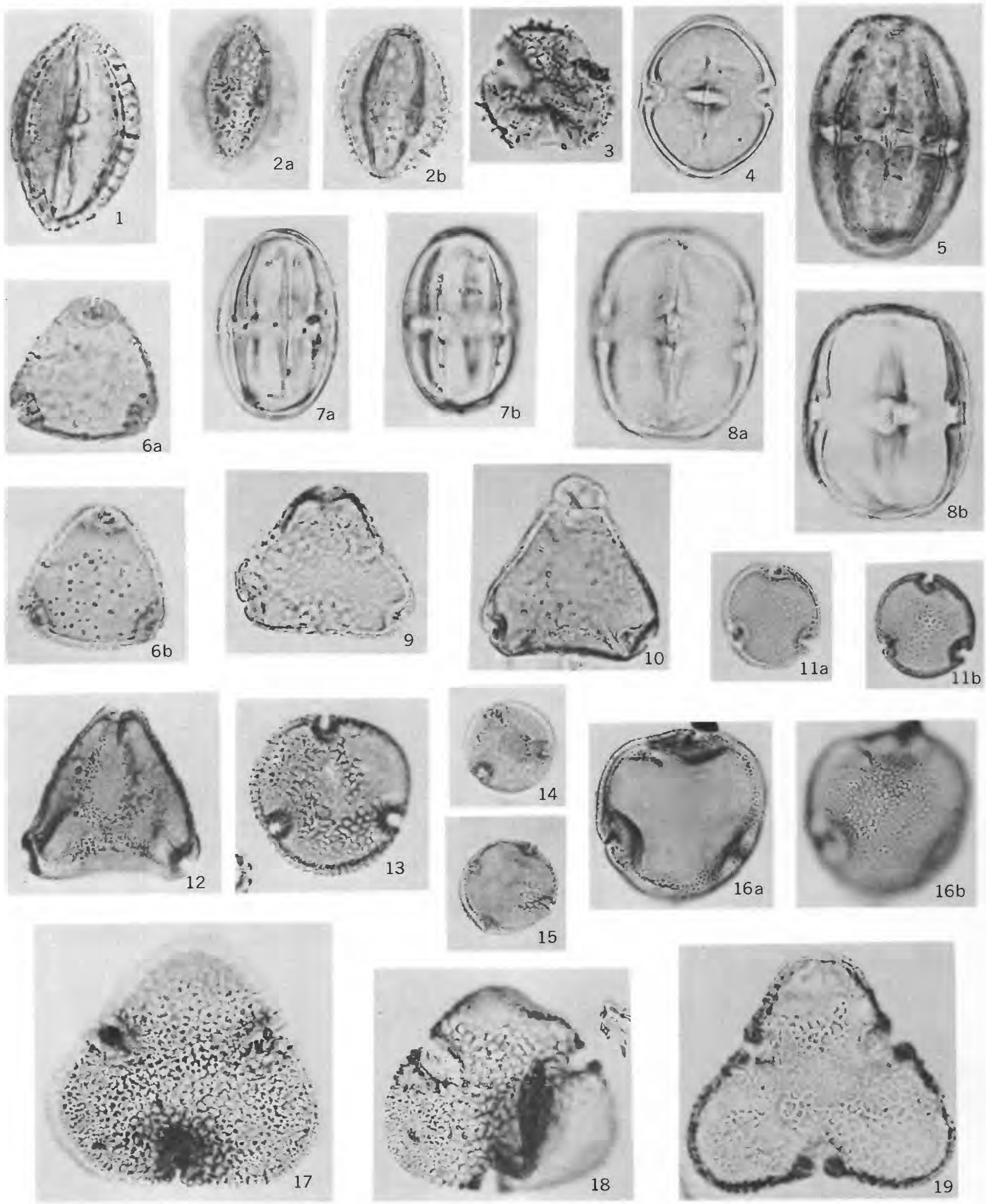

YAZOO POLLEN AND SPORES 
